Exploring a pathway for time-resolved studies of polymer fatigue related to nanostructure evolution

This article has been downloaded from IOPscience. Please scroll down to see the full text article.

2010 IOP Conf. Ser.: Mater. Sci. Eng. 14012010

(http://iopscience.iop.org/1757-899X/14/1/012010)

View the table of contents for this issue, or go to the journal homepage for more

Download details:

IP Address: 95.118.126.165

The article was downloaded on 26/02/2011 at 19:22

Please note that terms and conditions apply. 


\title{
Exploring a pathway for time-resolved studies of polymer fatigue related to nanostructure evolution
}

\author{
Ahmad Zeinolebadi and Norbert Stribeck \\ University of Hamburg, Dept. of Chemistry, Inst. of Technical and Macromolecular \\ Chemistry, Bundesstr. 45, 20146 Hamburg, Germany \\ E-mail: Norbert.Stribeck@desy.de
}

\begin{abstract}
An uniaxially oriented polymer blend based on high-density polyethylene (HDPE) that is reinforced by polyamide 6 microfibrils is subjected to slow mechanical fatigue tests. Simultaneously, the nanostructure evolution is monitored by two-dimensional (2D) patterns of the small-angle X-ray scattering (SAXS). The objective is to demonstrate that subtle changes of macroscopic strain and nanostructure parameters can be recorded with an accuracy that is sufficient to detect mechanisms that relate fatigue and nanostructure variation.

The macroscopic deformation of the material is recorded by a video camera and the captured pictures are analyzed by automated image processing methods to compute the macroscopic strain. From the 2D patterns the multidimensional chord distribution function (CDF) is calculated, and the scattering power $Q$ is determined. Peaks of the CDF that are related to the semicrystalline domain structure inside the HDPE are identified. The position of their centers of gravity is tracked automatically from the voluminous series of scattering patterns. The resulting curves demonstrate the relation between mechanical load and nanostructure evolution.
\end{abstract}

\section{Introduction}

Microfibrillar reinforced composites [1-6] (MFC) are a class of composite materials in which both the matrix and the reinforcing phase are composed of polymers. In this class of composites the microfibrillar structure of the reinforcing polymer within the polymer matrix is formed in situ during processing. Two immiscible polymers with different melting points are melt-blended. The main part is made from the lowermelting polymer. It is going to become the matrix, whereas the other polymer is going to become the microfibrillar reinforcement. Subsequent cold-drawing turns the domains of the minor part into microfibrils. Final processing (injection molding, extrusion, etc.) of this precursor is done at a temperature between the melting points of the two components. Hence, only the matrix polymer is isotropized. One major advantage of these materials is that they contain no mineral additives. Thus, after burning their ash content is low. This meets the future European legislation for materials to be used in automotive industries. In addition, tough MFCs are light-weight and suited to replace metal in cars. Therefore, there is an increasing demand for this class of materials in industry.

In many practical applications materials are subjected to dynamic or cyclic loadings. Hence, resistance [7] to dynamic load (i.e. low fatigue [8-10]) is required. There is abundant experimental literature on the relation between materials structure and mechanical load [11]. Nevertheless, the number of papers in which scattering is studied simultaneously during fatigue tests is still small [12,13]. Fortunately, recent progress at synchrotron x-ray radiation facilities provides the possibility to follow the variation of anisotropic scattering patterns of polymers during mechanical tests with sufficient accuracy.

However, a major challenge of time-resolved x-ray scattering experiments is the huge number of recorded patterns that amounts to hundreds or even thousands. Thus, one has to be either content with the basic information available from qualitative analysis of the patterns - which can be though time consuming and even confusing - or one should look for a fast automated method. A programming environment that is 
adapted to processing of multidimensional data and rapid prototyping such as PV-WAVE ${ }^{\circledR}[14]$ provides the chance to develop fast automatic algorithms for the processing of anisotropic scattering patterns. Ultimately, structural parameters can be computed automatically. Utilizing such an environment the data evaluation effort shrinks to the time spent during the experiment.

In ongoing work we investigate various precursors of microfibrillar reinforced composites in which the (polyethylene) matrix is not yet isotropized. Here we report first results on one of the samples that demonstrate the power of the method. The studied materials are considered sufficiently representative for other thermoplastic polymers that shall be subjected to load-cycling tests in order to shed more light on fatigue mechanisms as a function of materials composition.

\section{Experimental}

\subsection{Material}

The studied MFC material is made from 80 wt.- $\%$ high-density polyethylene (HDPE) and 20 wt.- $\%$ polyamide 6 (PA6). It is one sample from a series in which the composition of 4 components has been varied. Details concerning the principle of the preparation [6], the design of the extruder line [15], and the making of the studied material [16] have been published elsewhere.

\subsection{Mechanical tests}

Cyclic loading in the X-ray beam is performed by a self-made extensometer. The machine performs symmetric drawing. Signals from load cell and transducer are recorded during the experiment. After approaching different pre-strains (ca. 6\% and ca. 10\% engineering strain) the samples are cycled between two fixed distances of the cross-heads. Thus, strain-controlled load-cycling instead of stress-controlled cycling is performed. In the experiments the strain rate, $\varepsilon$, is close to $\pm 1.5 \times 10^{-4} s^{-1}$. During the tests a video camera monitors the sample and captures 2 pictures per cycle of the X-ray detector. Determination of the local macroscopic strain, $\varepsilon$, is based on the recorded video frames (Fig. 1).The required precision is obtained by computing the "correlation function" of the fiducial-mark structure on the fiber and fitting the resulting "long-period peak". The method has recently been published [16]. Most of the materials from the series show little fatigue and less variation of the scattering, except for the one that is presented here in this paper.

\subsection{X-ray scattering}

SAXS is performed at the synchrotron beamline A2 at HASYLAB, Hamburg, Germany. The wavelength of the X-ray beam is $0.15 \mathrm{~nm}$, and the sample-detector distance is $2542 \mathrm{~mm}$. Scattering patterns are collected by a 2D detector (marccd 165; mar research, Norderstedt, Germany) operated in $1024 \times 1024$ pixel mode (pixel size: $158.2 \times 158.2 \mu 5^{2}$ ). Thus, for the typical long period of $20 \mathrm{~nm}$ a variation of the peak position by one pixel causes a long-period variation of below $1 \%$. During the deformation experiments, scattering patterns are recorded every $30 \mathrm{~s}$ with an exposure of $23 \mathrm{~s}$.

\section{Data evaluation}

The basic data evaluation steps are documented in a text book [17]. The scattering patterns are normalized to the flux of the incident primary beam. Intensity loss due to absorption in the sample is compensated using the measured intensities of the primary beam before and after the sample, respectively. The machine background is subtracted. Pixels in the shade of the beam stop and outside the bordering vacuum tube are marked invalid. The fiber pattern is centered and aligned. Part of the invalid regions can be filled from symmetry 


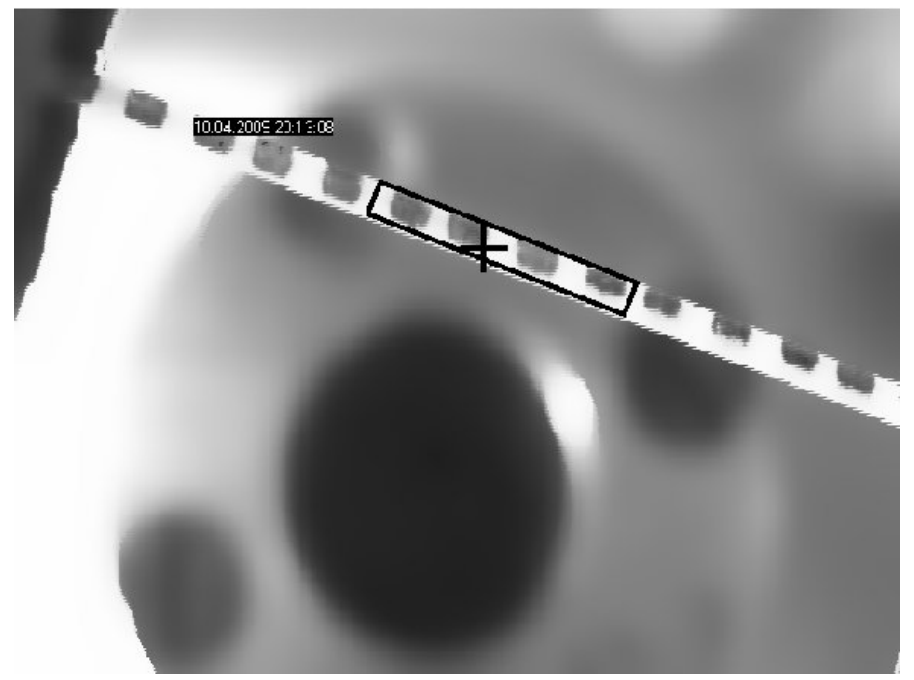

Figure 1: One video frame taken from a series that is used to determine the local macroscopic strain $\varepsilon$. The fiber carries fiducial marks. The cross indicates the position of the center of the primary beam. The box is the ROI (region of interest). From the image inside the ROI the correlation function is computed. Its longperiod peak varies with $\varepsilon$, as long as the fiber remains taut

consideration. The remnant central blind hole is filled applying a stiff parabolic extrapolation [18]. The pattern is projected on the representative fiber plane. Multiplication by $s^{2}$ applies the real-space Laplacian. The density fluctuation background is determined by low-pass filtering. It is eliminated by subtraction. The resulting interference function, $G\left(s_{12}, s_{3}\right)$, describes the ideal multiphase system. Its $2 \mathrm{D}$ Fourier transform is the chord distribution function $(\mathrm{CDF}), z\left(r_{12}, r_{3}\right)$. From $G\left(s_{12}, s_{3}\right)$ the scattering intensity $I_{i d}\left(s_{12}, s_{3}\right)$ of the ideal multiphase system can be reconstructed. From this pattern the well-known scattering power

$$
Q=\iiint I_{i d}(s) d^{3} s
$$

is computed. $Q$ is already normalized with respect to the irradiated volume, because of the respective normalization of the measured intensity. For this normalization we have taken advantage of the fact that the cross-section of the primary beam is oblong with its long axis ( $3 \mathrm{~mm})$ perpendicular to the fiber. Thus, the taut fiber sample is totally immersed in the primary beam. Now assuming incompressibility, a quantity that is proportional to the irradiated volume can be estimated from the initial diameter of the fiber and the actual macroscopic strain, $\varepsilon(t) . t$ is the time elapsed during the experiment. Because the major fraction of the studied materials is made from semicrystalline polyethylene, a major contribution [17,19-22] to the invariant [22]

$$
Q=v(1-v)\left(\rho_{c}-\rho_{a}\right)^{2}+X
$$

originates from the two-phase structure of the polyethylene with $v$ being its volume crystallinity, and $\rho_{c}-\rho_{a}$ the contrast between the crystalline and the amorphous electron density. The unknown quantity $X$ is predominantly originating from the embedded reinforcing needle-shaped domains (PA6), and from voids.

In addition, the nanoscopic strain, $\varepsilon_{\text {nano }}$, is estimated from the variation of the long period peaks in either the scattering pattern or in the corresponding CDF. In order to increase the accuracy of determination, the peaks are fitted by bivariate polynomials, and the parameters of the peak fit are transformed into high-resolution nanostructure parameters (long period, average domain extension, 

width of domain-thickness distribution) [16].

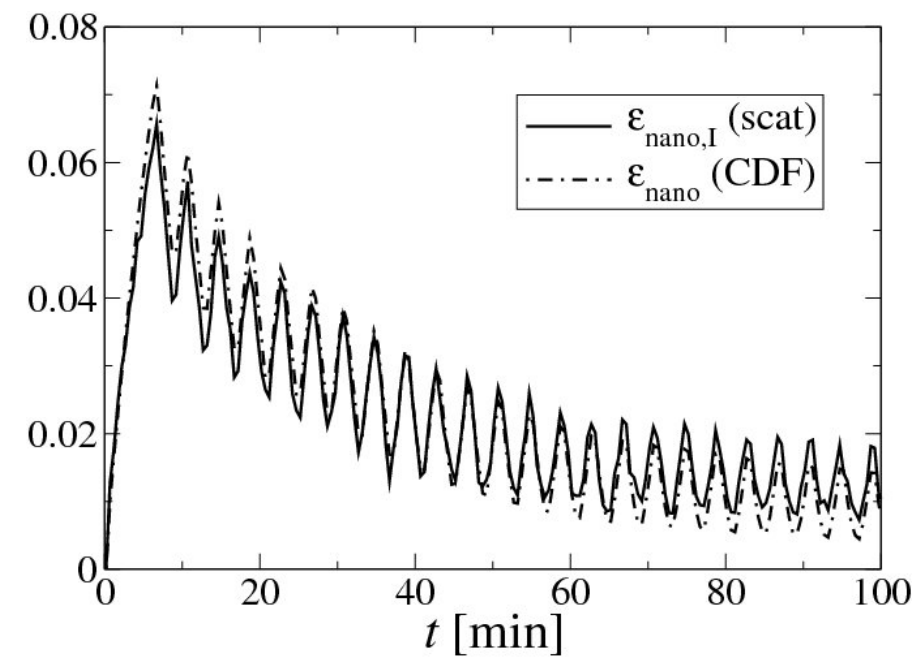

Figure 2: Comparison of nanoscopic strains $\varepsilon_{\text {nano,I }}$ and $\varepsilon_{\text {nano }}$, as determined from the scattering pattern $I\left(s_{12}, s_{3}\right)$ and from the CDF, $z\left(r_{12}, r_{3}\right)$, respectively. Material cycled about high pre-strain ("high-cycling experiment")

\section{Results and discussion}

The nanoscopic strains estimated from the scattering pattern, $\varepsilon_{\text {nano, } I}$, and from the $\mathrm{CDF}, \varepsilon_{\text {nano }}$, are compared in Fig. 2.The difference between the two methods is small. It is similar even for the other studied samples that are not in this paper. The observed systematic deviation can be explained by the different weighting of the long-period distribution in the scattering pattern and in the CDF. In the determination from the scattering pattern the long distances between the crystalline polyethylene domains are overestimated. A smaller systematic error may arise in the determination from the CDF, because we do not separate the series of distance distribution peaks by fitting. Instead, we simply search the peak maximum in the CDF. Consequently, heavily overlapping skewed distributions may lead to a systematic shift of the determined peak maximum.

Figure 3 shows recorded SAXS patterns and the corresponding CDFs for the material chosen. Obviously, the differences are subtle even though the picked images are selected from extreme states with respect to the sequence of load cycles. Figures 4 and 5 show the evolution of macroscopic and nanoscopic parameters for cycling about a low and a high pre-strain, respectively. In the case of low pre-strain the material shows weak fatigue only, whereas the same material subjected to a higher pre-strain shows very strong fatigue which can be easily inferred from the decrease of the stress, $\sigma$, from cycle to cycle. In Fig. 5 the macroscopic strain, $\varepsilon$, becomes negative after ca. 40 min. This observation may be explained by viscous flow of the material. During the further course of the experiment $(t>80 \mathrm{~min})$ this fatigue flow even causes the sample to become slack. It bends in each cycle close to the lower dead centers. As a result, the apparent $\varepsilon$ drops considerably. The reason is in the method of determination. As the fiducial-mark grating is tilted with respect to the original direction of the fiber by an angle $\alpha$, its lattice constant $L_{m}$ appears shortened to $L_{m} \cos \alpha$ with respect to the orientation of the ROI (cf. Fig. 1). Additionally, in this region an artificial drop of the invariant $Q$ is observed. The reason is of geometric nature, as well. Here the bent sample has moved out of the X-ray beam. As a result, the values of $Q(t>80 \mathrm{~min})$ cannot be discussed in terms of structure evolution. Similar is valid for $\varepsilon(t>80 \mathrm{~min})$ in the high-cycling experiment (Fig. 5).

No significant phase shift is observed between $\varepsilon_{\text {nano }}, \sigma$, and $\varepsilon$ both for low pre-strain and for high prestrain. Thus, these quantities predominantly reflect the forced oscillation of the cross-heads. On the other hand, for both pre-strains during the first cycles the nanoscopic strain, $\varepsilon_{\text {nano }}$, is considerably lower than the macroscopic strain, $\varepsilon$. This observation shows that initially the elastic modulus of the semicrystalline stacks is higher than that of the surrounding amorphous matrix. Later, as the macroscopic relaxation starts in the 


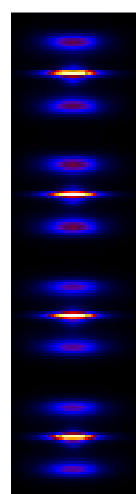

SAXS CDF

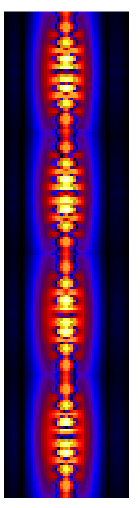

$\min \mathcal{E}$

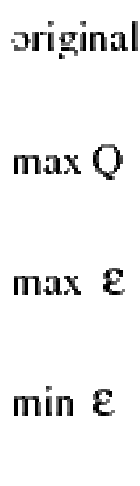

SAXS CDF
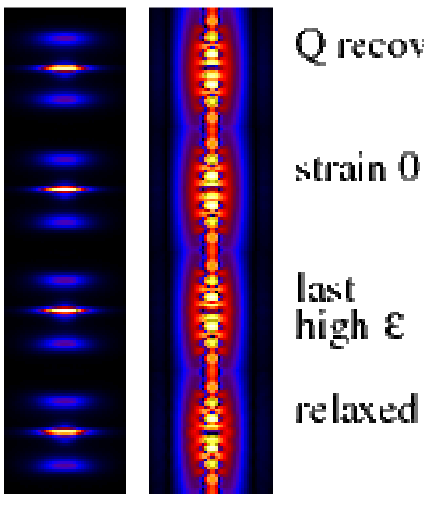

Inst

relaxed

Figure 3: Selected SAXS patterns $I\left(s_{12}, s_{3}\right)$ and CDFs $\left|z\left(r_{12}, r_{3}\right)\right|$ from the experiment in which the load-cycling has been performed on high pre-strain ("high-cycling experiment"). Intensities on logarithmic scale. Displayed regions: $-0.1 \mathrm{~nm}^{-1}<s_{12}, s_{3}<0.1 \mathrm{~nm}^{-1},-75 \mathrm{~nm}<r_{12}, r_{3}<75 \mathrm{~nm}$

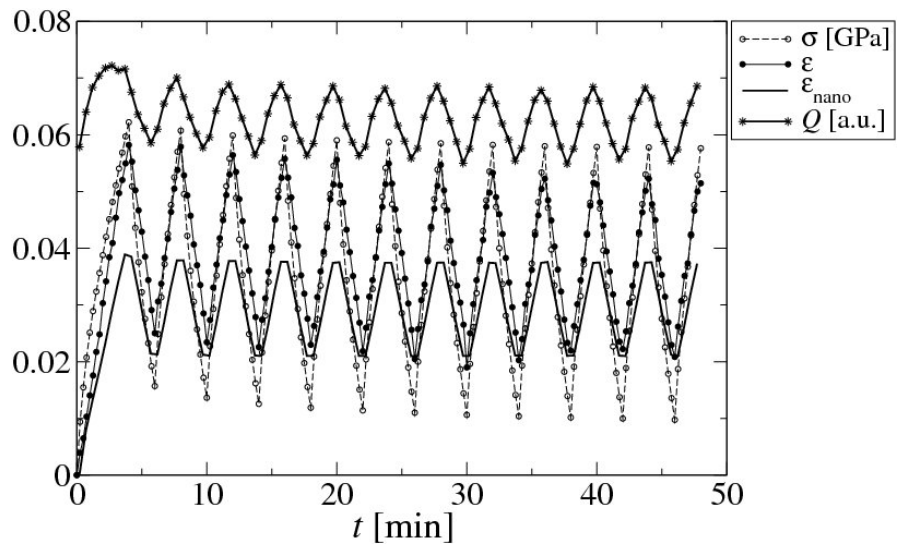

Figure 4: HDPE/PA6 (80/20) cycled about low pre-strain 


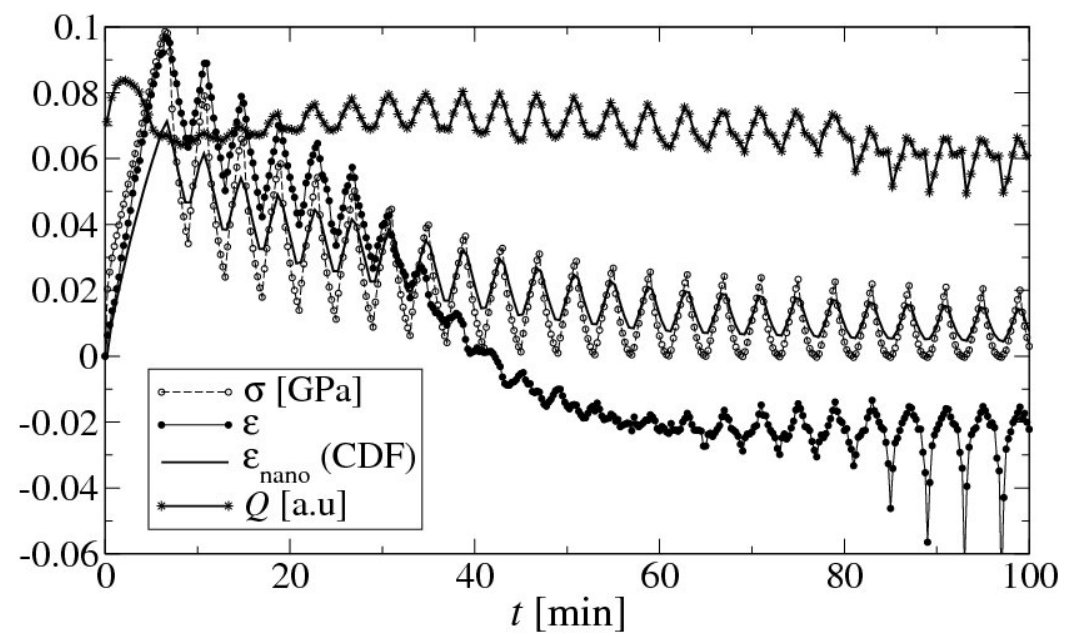

Figure 5: The material in the high-cycling experiment is showing considerable fatigue 
high-cycling material, the nanoscopic strain of the semicrystalline stacks is unaffected and keeps cycling. This shows that the viscous flow of the material during stress relaxation is predominantly taking place in the amorphous matrix that is far away from the embedded crystallites.

Because we are controlling the cross-head oscillation but not the stress oscillation, the average stress $\sigma$ is decreasing from cycle to cycle. For the low-cycling experiment the decrease rate is rather low, but high if the material is cycled about high pre-strain.

Compared to these parameters the evolution of the scattering power, $Q$, appears to be different. At the beginning of both experiments the scattering power is increasing. Eq. (2) shows that it is difficult to assign the reason for the increase of the integral quantity $Q$ to one of the structural parameters in the equation, unless one takes advantage of the anisotropy of the scattering patterns. Based on the integral quantity alone, it would not even be possible to decide if an increase or a decrease of crystallinity or contrast were the reason for a specific change of $Q$.

Nevertheless, even from a first visit to the data some heuristic assessments can be made. When in the low-cycling experiment (Fig. 4) the first top dead-center is reached ( $t=4 \mathrm{~min}), Q(t)$ has just passed a maximum. In the high-cycling experiment (Fig. 5) the first top dead-center is much later - at higher strain $\varepsilon$ and at higher stress $\sigma$. At this time, $Q(t)$ has already dropped considerably below the initial scattering power. In the following cycles an almost negligible $Q$-oscillation is considerably amplified by the external load, until its amplitude appears saturated from the 10th cycle. This is completely different in the low-cycling experiment (Fig. 4), where the amplitude of the $Q$-oscillation is high and constant from the first cycle to the last.

Comparison of these observations indicates that the initial increase of $Q$ is probably related to a fortification of the nanostructure, similar to the strain-induced crystallization that has been detected in an earlier study of hard-elastic polypropylene [23], whereas the subsequent drop of $Q$ appears to be indicative for structure deterioration. In a recent study [16] on continuous straining of the microfibrillar reinforced HDPE/PA-blends we have already carried out a detailed analysis of corresponding anisotropic scattering patterns yielding information on the evolution of crystal shape. Concerning the presented study, similar analysis of the presented time-resolved data is in progress.

Finally, it appears worth mentioning that in the high pre-strain load-cycling experiment the evolution of $Q(t)$ indicates some structural adaption process. The last part of the initial increasing load ramp appears to deteriorate the original nanostructure. Thereafter, the cycling appears to "teach" the material an adapted nanostructure that, again, responds more or less elastically on the forced external load cycles.

\section{Conclusions}

The results show that subtle changes of mechanical and nanostructural parameters can be recorded with sufficient precision in in situ time-resolved SAXS experiments. Fatigue behavior is clearly detected. Nevertheless, perturbing artifacts may show up in such experiments, which monitor short elongation as a result of sample bending due to fatigue relaxation. In order to further study the mechanisms of fatigue from the recorded data, the series of anisotropic scattering data must be analyzed in more depth with respect to nanostructure evolution.

The already existing bottleneck of data evaluation will become more severe, when we will be able to increase the strain rates after the start of PETRA III operation at the synchrotron in Hamburg. Nevertheless, with further improved data analysis tools and instrumentation detailed evolution data of macroscopic mechanical parameters and nanoscopic structural parameters from time-resolved scattering studies will become available. We believe that these results will shed new light upon the relation between mechanical properties and the structure of polymer materials. 
Acknowledgments. The authors thank the Hamburg Synchrotron Radiation Laboratory (HASYLAB) for beam time granted in the frame of project II-04-039. This work has been supported by the 7th framework program of the European Union (Project NANOTOUGH FP7-NMP-2007-LARGE). We gratefully acknowledge the preparation of the material by Z. Denchev, University of Miñho, Portugal.

\section{References}

[1] Evstatiev M and Fakirov S 1992 Polymer 33 877-880

[2] Evstatiev M, Nicolov N and Fakirov S 1996 Polymer 37 4455-4463

[3] Fakirov S, Stribeck N, Apostolov A A, Denchev Z, Krasteva B, Evstatiev M and Friedrich K 2001 J. Macromol. Sci. Phys. 40 935-957

[4] Schmidt P, Baldrian J, Ščudla J, Dybal J, Raab M and Eichhorn K J 2001 Polymer 41 5321-5326

[5] Li Z M, Yang M B, Feng J M, Yang W and Huang R 2002 Mater. Res. Bull. 37 21852197

[6] Denchev Z Z and Dencheva N V 2008 Polym. Inter. 57 11-22

[7] Thorby D 2008 Structural Dynamics and Vibration in Practice 1st ed (Amsterdam: Butterworth-Heinemann)

[8] Peterlin A 1972 Ann. Rev. Mater. Sci. 2 349-380

[9] Takemori M T 1984 Ann. Rev. Mater. Sci. 14 171-204

[10] Crist B 1995 Ann. Rev. Mater. Sci. 25 295-323

[11] Pegoretti A 2009 Nano- and Micro-Mechanics of Polymer Blends and Composites ed Karger-Kocsis J and Fakirov S (Munich: Hanser) chap 9, pp 301-339

[12] Brown H R, Kramer E J and Bubeck R A 1988 J. Mater. Sci. 23 248-252

[13] Toki S, Sics I, Burger C, Fang D, Liu L, Hsiao B S, Datta S and Tsou A H 2006 Macromolecules 39 3588-3597

[14] VNI 2007 Pv-wave manuals V 7.5, Houston, TX, USA

[15] Dencheva N, Oliveira M J, Carneiro O S, Pouzada A S and Denchev Z 2009 J. Appl. Polym. Sci. submitted

[16] Denchev Z, Dencheva N, Funari S S, Motovilin M, Schubert T and Stribeck N 2010 J. Polym. Sci. Part B: Polym. Phys. 48 237-250

[17] Stribeck N 2007 X-Ray Scattering of Soft Matter (Heidelberg, New York: Springer)

[18] Stribeck N and Nöchel U 2008 J. Appl. Cryst. 41 715-722

[19] Ran S, Zong X, Fang D, Hsiao B S, Chu B and Phillips R A 2001 Macromolecules 34 2569-2578

[20] Jánosi A 1983 Monatsh. f. Chemie 114 377-383

[21] Glatter O and Kratky O (eds) 1982 Small Angle X-ray Scattering (London: Academic Press)

[22] Baltá Calleja F J and Vonk C G 1989 X-Ray Scattering of Synthetic Polymers (Amsterdam: Elsevier)

[23] Stribeck N, Nöchel U, Funari S S, Schubert T and Timmann A 2008 Macromol. Chem. Phys. 209 1992-200 